11

\title{
Квадрупольное электромагнитное излучение осциллирующей заряженной капли
}

\author{
() А.И. Григорьев, Н.Ю. Колбнева, С.О. Ширяева \\ Ярославский государственный университет им. П.Г. Демидова, \\ 150000 Ярославль, Россия \\ e-mail: grig@uniyar.ac.ru
}

(Поступило в Редакцию 24 мая 2016 г. В окончательной редакции 25 августа 2016 г.)

Показано, что интенсивность электромагнитного излучения заряженной капли, обнаруживаемая в аналитических расчетах первого порядка малости по безразмерной амплитуде ее осцилляций, определена изменением во времени квадрупольного момента капли.

DOI: 10.21883/JTF.2017.06.44517.1901

\section{Введение}

Четверть века назад появился первый анализ электромагнитного излучения осциллирующей заряженной капли [1]. В последующие годы результаты работы [1] уточнялись [2] и обобщались [3]. Затем появилась работа [4], в которой было указано на расчетную ошибку в [1-3]: в этих работах были использованы неправильные асимптотики цилиндрических функций Бесселя третьего рода при малых значениях аргумента. В работе [5] ошибка, допущенная в [1-3], была исправлена. Но еще в [6] было предположено, что излучение, обнаруживаемое в теоретических расчетах первого порядка малости по амплитуде осцилляций [1-3], является квадрупольным, поскольку дипольное излучение в указанном порядке малости не может быть обнаружено. Однако это предположение не было подтверждено прямым расчетом.

Сама задача о расчете интенсивности излучения от осциллирующей капли представляет интерес в связи с актуальностью проблемы пассивной радиолокации метеорологических объектов различных типов [7,8].

Капли, входящие в состав облаков различных типов, туманов и смогов [9], находятся в приземном электростатическом поле. Весьма часто такие капли несут и электрический заряд [9-11]. В жидкости же всегда существует капиллярное волновое движение, порождаемое уже тепловым движением молекул жидкости, и имеет место волновое искажение равновесной сферической формы капель. Характерная амплитуда такого волнового искажения $\xi \sim \sqrt{\kappa T / \sigma}$, где $\kappa=8.31 \cdot 10^{7} \mathrm{erg} /(\mathrm{mol} \cdot \mathrm{K})-$ постоянная Больцмана, $T$ - абсолютная температура в Кельвинах [12], $\sigma$ - коэффициент поверхностного натяжения жидкости. При температурах порядка комнатной амплитуда тепловых колебаний для всех жидкостей меньше ангстрема. Однако это дает основание утверждать, что свободная поверхность капель непрерывно осциллирует. Под влиянием внешних силовых воздействий амплитуда осцилляций может расти и достигать десятков процентов от радиусов капель [13-15]. Для заряженных капель осцилляции их поверхности приводят к генерации электромагнитного излучения.
Аналитическому решению указанной задачи и посвящена настоящая работа.

\section{Физическая постановка задачи}

Рассмотрим задачу об электромагнитном излучении равновесной сферической с радиусом $R$ формы поверхности капли идеальной, несжимаемой, идеально проводящей жидкости с плотностью $\rho$, коэффициентом поверхностного натяжения $\sigma$. Примем, что капля находится в вакууме, ее полный заряд равен $Q$. Заряд распределяется по возмущенной капиллярным волновым движением поверхности капли.

Интенсивность полного электромагнитного излучения для ускоренно движущейся системы зарядов состоит из трех независимых частей: дипольной, квадрупольной и магнитно-дипольной [16]:

$$
\begin{aligned}
I & =\frac{2}{3 c^{3}}\left(\frac{\partial^{2}}{\partial t^{2}} \mathbf{d}\right)^{2}+\frac{1}{180 c^{5}} \sum_{\alpha, \beta=1}^{3}\left(\frac{\partial^{3}}{\partial t^{3}} D_{\alpha \beta}\right)^{2} \\
& +\frac{2}{3 c^{3}}\left(\frac{\partial^{2}}{\partial t^{2}} \mathbf{m}\right)^{2} .
\end{aligned}
$$

где $\mathbf{d}=Q \mathbf{R}_{q}$ - дипольный момент, $D_{\alpha \beta}-$ тензор квадрупольного момента (характеризует симметрию в расположении зарядов [17] $), \mathbf{m}=\frac{Q}{2 c}\left(\mathbf{R}_{q} \times \partial \mathbf{R}_{q}\right)-$ магнитный момент, $\mathbf{R}_{q}$ - радиус-вектор, характеризующий положение центра „эффективного“ заряда всей системы зарядов относительно выбранного начала отсчета. В рассматриваемом случае заряженной капли, на поверхности которой реализуются осесимметричные осцилляции, естественно принять, что $\mathbf{R}_{q}$ характеризует положение центра „эффективного“ заряда капли относительно центра капли. Легко показать, что в расчетах первого порядка малости по амплитуде осцилляций центр капли совпадает с ее центром масс.

Из трех компонент излучения в формуле (1) самая интенсивная первая (дипольная), вторая и третья (квадрупольная и магнитно-дипольная) имеют одинаковый порядок величины. 
Несложно показать, что в расчетах первого порядка малости по безразмерной амплитуде осцилляций поверхности сферической капли смещение „эффективного“ заряда отсутствует $\mathbf{R}_{q}=0$. Следовательно, дипольный и магнитный моменты равны нулю $\mathbf{d}=\mathbf{m}=0$, а дипольное и магнитно-дипольное излучения отсутствуют. Таким образом, в расчетах первого порядка малости интенсивность электромагнитного излучения в (1) определяется только квадрупольной компонентой, где тензор квадрупольного момента $D_{\alpha \beta}(t)$ определяется выражением

$$
D_{\alpha \beta}(t)=\iiint_{V} \gamma(\mathbf{r}, t)\left(3 x_{\alpha}(t) x_{\beta}(t)-\delta_{\alpha \beta} r^{2}(t)\right) d V,
$$

где $\gamma(\mathbf{r}, t)$ - объемная плотность заряда, $\delta_{\alpha \beta}$ - дельтасимвол Кронекера $x_{\alpha}(t)$ и $x_{\beta}(t)$ - координаты радиусвектора $\mathbf{r}(t)$ в декартовой системе координат для точки, лежащей в объеме капли $V$.

При сферически симметричном распределении заряда квадрупольный электрический момент равен нулю [17] как для постоянного объемного заряда (заряженный диэлектрический шар) [16], так и для поверхностного заряда (идеально проводящая заряженная сфера) [18]. В частности, наличие отличного от нуля квадрупольного момента у атомного ядра свидетельствует об отличии его формы от сферической ([17]).

Все расчеты задачи будем проводить в сферической системе координат $(r, \theta, \varphi)$ с началом в центре масс капли в безразмерных переменных, в которых $R=\rho=\sigma=1$.

Сформулируем задачу об электромагнитном квадрупольном излучении заряженной сферической капли при малых осцилляциях ее поверхности.

\section{Математическая формулировка задачи}

Примем, что равновесная сферическая поверхность идеально проводящей заряженной сферической капли претерпевает тепловое возмущение $\xi(\theta, t)$ амплитуды $\varepsilon$, существенно меньшей радиуса капли. Ограничим рассмотрение осесимметричными возмущениями (что не снижает общности рассмотрения, но существенно уменьшает громоздкость).

Уравнение, описывающее поверхность капли, в сферической системе координат с началом в центре масс капли в безразмерных переменных имеет вид

$$
r(\theta, t)=1+\xi(\theta, t), \quad|\xi| \ll 1 .
$$

Движение жидкости в капле будем полагать потенциальным и примем, что поле скоростей движения жидкости в капле $\mathbf{V}(\mathbf{r}, t)=\nabla \psi(\mathbf{r}, t)$ полностью определяется потенциалом скорости $\psi(\mathbf{r}, t)$. Естественно принять, что поле скоростей волнового течения жидкости имеет тот же порядок малости, что и амплитуда осцилляций поверхности капли $\psi(\mathbf{r}, t) \sim \xi(\theta, t) \sim \varepsilon$.
Будем полагать, что электрическое поле в окрестности капли создается ее зарядом. Это поле будем характеризовать напряженностью $\mathbf{E}(\mathbf{r}, t)$, связанной с электрическим потенциалом $\Phi(\mathbf{r}, t)$ известным соотношением $\mathbf{E}(\mathbf{r}, t)=-\Phi(\mathbf{r}, t)$.

Тогда математическая формулировка задачи об электромагнитном квадрупольном излучении заряженной капли примет вид:

$$
\begin{gathered}
\Delta \psi(\mathbf{r}, t)=0, \\
\Delta \Phi(\mathbf{r}, t)=0, \\
r \rightarrow 0: \quad \psi(\mathbf{r}, t) \rightarrow 0, \quad r \rightarrow \infty: \quad \Phi(\mathbf{r}, t) \rightarrow 0, \\
r=1+\xi(\theta, t): \frac{\partial \xi(\theta, t)}{\partial t}=\frac{\partial \psi(\mathbf{r}, t)}{\partial r}-\frac{1}{r^{2}} \frac{\partial \psi(\mathbf{r}, t)}{\partial \theta} \frac{\partial \xi(\theta, t)}{\partial \theta}, \\
P-P_{\mathrm{atm}}+P_{q}=P_{\sigma}, \quad \Phi(\mathbf{r}, t)=\Phi_{S}(t) .
\end{gathered}
$$

Дополним выписанную систему условиями: неизменности полного объема капли (следствие несжимаемости жидкости), неподвижности ее центра масс, и сохранения полного заряда капли.

$$
\begin{gathered}
\int_{V} r^{2} d r \sin \theta d \theta d \varphi=\frac{4}{3} \pi, \int_{V} \mathbf{r} r^{2} d r \sin \theta d \theta d \varphi=0 \\
V=[0 \leq r \leq 1+\xi(\theta, t) ; 0 \leq \theta \leq \pi ; 0 \leq \varphi \leq 2 \pi], \\
\frac{1}{4 \pi} \oint_{S}(\mathbf{n}, \nabla \Phi) d S=Q, \\
S=[r=1+\xi(\theta, t), 0 \leq \theta \leq \pi, 0 \leq \varphi \leq 2 \pi] .
\end{gathered}
$$

В выражениях (7), (8) введены следующие обозначения: $\Phi_{S}(t)$ - постоянное вдоль поверхности капли значение ее электрического потенциала, $P=P_{0}-\frac{\partial \psi(\mathbf{r}, t)}{\partial t}-$ гидродинамическое давление, $P_{0}-$ постоянное давление внутри капли в состоянии равновесия, $P_{\mathrm{atm}}-$ постоянное давление вне капли, $P_{q}=(\nabla \Phi(\mathbf{r}, t))^{2} / 8 \pi-$ давление электрического поля, $P_{\sigma}=\operatorname{div} \mathbf{n}(\mathbf{r}, t)-$ капиллярное давление, $\mathbf{n}(\mathbf{r}, t)$ - единичный вектор нормали, определяемый в виде:

$$
\mathbf{n}(\theta, t)=\left.\frac{\nabla(r-r(\theta, t))}{|\nabla(r-r(\theta, t))|}\right|_{r=r(\theta, t)}=\mathbf{e}_{r}-\frac{\partial \xi(\theta, t)}{\partial \theta} \mathbf{e}_{\theta},
$$

где $\mathbf{e}_{r}$ и $\mathbf{e}_{\theta}-$ орты сферической системы координат.

Искомые величины разложим по порядкам малости безразмерной амплитуды осцилляций $\varepsilon$ :

$$
\begin{gathered}
\xi(\theta, t)=\xi^{(1)}(\theta, t)+O\left(\varepsilon^{2}\right), \psi(\mathbf{r}, t)=\psi^{(1)}(\mathbf{r}, t)+O\left(\varepsilon^{2}\right), \\
P(\mathbf{r}, t)=P^{(0)}(\mathbf{r})+P^{(1)}(\mathbf{r}, t)+O\left(\varepsilon^{2}\right) \\
P_{\sigma}(\mathbf{r}, t)=P_{\sigma}^{(0)}(\mathbf{r})+P_{\sigma}^{(1)}(\mathbf{r}, t)+O\left(\varepsilon^{2}\right) \\
P_{q}(\mathbf{r}, t)=P_{q}^{(0)}(\mathbf{r})+P_{q}^{(1)}(\mathbf{r}, t)+O\left(\varepsilon^{2}\right)
\end{gathered}
$$




$$
\Phi(\mathbf{r}, t)=\Phi^{(0)}(\mathbf{r})+\Phi^{(1)}(\mathbf{r}, t)+O\left(\varepsilon^{2}\right),
$$

где $\Phi^{(0)}(r)=\frac{Q}{r}$ - электрический потенциал в окрестности равновесной заряженной сферы, $\Phi^{(1)}(r, \theta, t)-$ добавка первого порядка малости к электрическому потенциалу, связанная с возмущением поверхности заряженной капли. Верхний индекс, стоящий в скобках, означает порядок малости по $\varepsilon$.

Подставляя разложения (10) в (4)-(8), выделим отдельно задачу первого порядка по $\varepsilon$ для нахождения возмущения формы поверхности капли $\xi(\theta, t)$ и поправки к электрическому потенциалу $\Phi^{(1)}(r, \theta, t)$ в окрестности возмущенной заряженной капли.

Решения уравнений (4), (5) для $\psi(\mathbf{r}, t)$ и $\Phi^{(1)}(\mathbf{r}, t)$, удовлетворяющие граничным условиям (6), а также и возмущение равновесной формы поверхности капли $\xi(\theta, t)$, запишем в виде рядов по осесимметричным полиномам Лежандра [19]:

$$
\begin{gathered}
\psi(r, \theta, t)=\varepsilon \sum_{n=0}^{\infty} D_{n}(t) r^{n} P_{n}(\mu), \quad \mu \equiv \cos \theta \\
\Phi^{(1)}(r, \theta, t)=\varepsilon \sum_{n=0}^{\infty} F_{n}(t) r^{-(n+1)} P_{n}(\mu), \\
\xi(\theta, t)=\varepsilon \sum_{n=0}^{\infty} M_{n}(t) P_{n}(\mu),
\end{gathered}
$$

где на порядок малости указывает степень $\varepsilon$.

\section{Задача первого порядка малости по $\varepsilon$}

В первом порядке малости по $\varepsilon$ для определения коэффициентов $D_{n}, F_{n}, M_{n}$ в решениях (11)-(13) из уравнений (7), (8) будем иметь систему:

$$
\begin{aligned}
& r=1: \quad \frac{\partial \xi(\theta, t)}{\partial t}=\frac{\partial \psi(r, \theta, t)}{\partial r}, \\
& -\frac{\partial \psi(r, \theta, t)}{\partial t}+\frac{1}{4 \pi} \frac{\partial \Phi^{(0)}(r)}{\partial r} \\
& \times\left(\frac{\partial \Phi^{(1)}(r, \theta, t)}{\partial r}+\frac{\partial^{2} \Phi^{(0)}(r)}{\partial r^{2}} \xi(\theta, t)\right) \\
& =-\left(2+L_{0}\right) \xi(\theta, t) \text {, } \\
& \Phi^{(1)}(r, \theta, t)+\frac{\partial \Phi^{(0)}(r)}{\partial r} \xi(\theta, t)=\Phi_{S}^{(1)}(t), \\
& \int_{0}^{\pi} \xi(\theta, t) \sin \theta d \theta=0, \quad \int_{0}^{2 \pi} \int_{0}^{\pi} \xi(\theta, t) \cos \theta d \theta=0 \\
& \int_{0}^{\pi}\left[\frac{\partial \Phi^{(1)}(r, \theta, t)}{\partial r}+\left(\frac{\partial^{2} \Phi^{(0)}(r)}{\partial r^{2}}+2 \frac{\partial \Phi^{(0)}(r)}{\partial r}\right) \xi(\theta, t)\right] \\
& \times \sin \theta d \theta=0,
\end{aligned}
$$

$$
L_{\theta} \equiv \frac{1}{\sin \theta} \frac{\partial}{\partial \theta}\left(\sin \theta \frac{\partial}{\partial \theta}\right) \text {. }
$$

Здесь $\Phi_{S}^{(1)}(t)$ - поправка первого порядка малости к величине потенциала поверхности капли.

Система (14) позволяет получить следующие соотношения для коэффициентов:

$$
\begin{array}{r}
M_{0}(t)=0, \quad M_{1}(t)=0, \quad D_{n}(t)=\frac{1}{n} \frac{\partial M_{n}(t)}{\partial t}, \quad(n \geq 2), \\
F_{n}(t)=Q M_{n}(t), \quad(n \geq 2), \quad \Phi_{S}^{(1)}=0 . \quad(15)
\end{array}
$$

Подставив коэффициенты $F_{n}(t)$ из $(15)$ в (12), получим выражение для поправки к электрическому потенциалу в окрестности возмущенной заряженной капли в виде:

$$
\Phi^{(1)}(r, \theta, t)=\varepsilon Q \sum_{n=2}^{\infty} M_{n}(t) r^{-(n+1)} P_{n}(\mu) .
$$

Для нахождения коэффициентов $M_{n}(t)$ при $n \geq 2$ из системы (14) получается однородное дифференциальное уравнение

$$
\frac{\partial^{2}}{\partial t^{2}} M_{n}(t)+\omega_{n}^{2} M_{n}(t)=0
$$

где частота собственных осцилляций заряженной сферической капли $\omega_{n}$ определяется следующим дисперсионным уравнением:

$$
\omega_{n}^{2}=n(n-1)((n+2)-W), \quad W=\frac{Q^{2}}{4 \pi},
$$

Решением уравнения (17) будут гармонические функции времени $t$ :

$$
M_{n}(t)=a_{n} \exp \left(i\left(\omega_{n} t+b_{n}\right)\right)+\text { c.c, } \quad(n \geq 2),
$$

где $a_{n}$ и $b_{n}$ - вещественные константы, определяемые из начальных условий, аббревиатура „с.с.“ означает слагаемые, комплексно сопряженные к выписанным.

В итоге, используя (3) и (13) для формы поверхности колеблющейся заряженной капли, запишем аналитическое выражение

$$
r(\theta, t)=1+\varepsilon \sum_{n=2}^{\infty} M_{n}(t) P_{n}(\mu),
$$

в котором амплитудные коэффициенты $M_{n}(t)$ определяются (19).

Поверхностную плотность заряда на идеально проводящей капле найдем, отталкиваясь от выражения потенциала (10) с учетом (16) и выражения для вектора нормали (9) с учетом (13) и (19), по известной формуле [20]:

$$
\begin{aligned}
v(\theta, t) & =\left.\frac{E_{n}(\mathbf{r}, t)}{4 \pi}\right|_{r=r(\theta, t)}=-\left.\frac{n(\mathbf{r}, t) \nabla \Phi(\mathbf{r}, t)}{4 \pi}\right|_{r=r(\theta, t)} \\
& =\frac{Q}{4 \pi}\left[1+\varepsilon \sum_{n=2}^{\infty}(n-1) M_{n}(t) P_{n}(\mu)\right] .
\end{aligned}
$$




\section{Излучение электромагнитных волн}

Для вычисления интенсивности электромагнитного излучения от капли рассчитаем тензор ее квадрупольного момента $D_{\alpha \beta}(t)$ в сферической системе координат с началом в центре масс капли, учитывая формальное соотношение, связывающее объемную плотность заряда $\gamma=\gamma(\mathbf{r}, t)$ с поверхностной на проводящей осесимметричной капле $v(\theta, t)$ :

$$
\gamma(r, \theta, t) \equiv v(\theta, t) \delta(r-r(\theta, t))
$$

где $\delta(r-r(\theta, t))$ - дельта-функция Дирака [17] в сферической системе координат с началом в центре капли. Наличие дельта функции в вышезаписанных выражениях превратит интегрирование по объему в (2) в интегрирование по возмущенной поверхности капли. В результате компоненты тензора электрического квадрупольного момента в первом приближении по безразмерной амплитуде осцилляций запишутся в виде

$$
\begin{aligned}
D_{12}= & D_{21}=D_{13}=D_{31}=D_{23}=D_{32}=0 \\
D_{11}(t) & =D_{22}(t)=-\frac{1}{2} D_{33}(t) \\
& =-\frac{1}{4} \int_{0}^{\pi}(\mathbf{n}(\theta, t), \nabla \Phi(\mathbf{r}, t)) r^{4}(\theta, t) \\
& \times\left(1-3 \cos ^{2} \theta\right) \sin \theta d \theta .
\end{aligned}
$$

Используя разложение для электрического потенциала (10) и вектор нормали (9) к возмущенной поверхности капли (20), определим подынтегральное выражение в $(22)$ с точностью до слагаемых $\sim \varepsilon$ :

$$
\begin{aligned}
& (\mathbf{n}(\theta, t), \nabla \Phi(\mathbf{r}, t)) r^{4}(\theta, t) \\
& =-\left(Q+2 Q \xi(\theta, t)-\frac{\partial \Phi^{(1)}(r, \theta, t)}{\partial r}\right) \\
& =-Q\left(1+\varepsilon \sum_{n=2}^{\infty}(n+3) M_{n}(t) P_{n}(\mu)\right) .
\end{aligned}
$$

Подставив (23) в (22) и учитывая условие ортогональности полиномов Лежандра, получим

$$
\begin{aligned}
D_{11}(t) & =D_{22}(t)=-\frac{1}{2} D_{33}(t) \\
& =-\frac{Q}{2} \int_{0}^{\pi}\left(P_{0}(\mu)+\varepsilon \sum_{n=2}^{\infty}(n+3) M_{n}(t) P_{n}(\mu)\right) \\
& \times P_{2}(\mu) \sin \theta d \theta \equiv-Q \varepsilon M_{2}(t) .
\end{aligned}
$$

Из этого решения следует, что квадрупольный момент сферической возмущенной капли и соответственно квадрупольное излучение определяются только второй модой осцилляций. Излучение, связанное с осцилляциями более высоких, чем вторая, мод заряженной капли, определится более высокими, чем квадрупольный, мультипольными моментами [17]. Однако интенсивность такого электромагнитного излучения будет существенно ниже (на много порядков), чем квадрупольное. Так, в [17] прямыми расчетами показано, что при увеличении номера моды осцилляций поверхности заряженной капли на единицу интенсивность излучения, связанного с этой модой, уменьшается на 14-15 порядков. Так, интенсивность октупольного излучения будет на 14-15 порядков слабее интенсивности квадрупольного. Интенсивность излучения следующего по порядку мультипольного момента, в свою очередь, будет на 14-15 порядков слабее октупольного. Подчеркнем, что речь идет о расчетах излучения в линейном приближении по безразмерной амплитуде осцилляций.

Следует отметить, что, рассматривая электрическое поле заряженной капли радиуса $R$ в точке $A$ на расстоянии $L \gg R$, электрическое поле можно разложить на компоненты по степеням отношения $R / L$, и таким образом получить дипольное поле, квадрупольное, октупольное и т.д. Принимая, что заряженная капля осциллирует с амплитудой $\xi$, где $R \gg \xi$, получаем второй малый параметр, по которому можно провести разложение. В вышеизложенном рассмотрении мы ограничились первой степенью $\xi / R$, но по $R / L$ мы взяли квадратичное разложение (квадрупольная составляющая). Третья степень разложения по $R / L$ даст октупольную компоненту. Дипольную компоненту поля мы не учитывали, так как в линейном по $\xi / R$ приближении дипольный момент равен нулю.

Для того чтобы получить численные оценки интенсивности излучения, зададим начальные условия в виде начальной деформации равновесной сферической формы капли и равенства нулю начальной скорости движения ее поверхности

$$
t=0: \xi(0)=\sum_{j \in \Xi} h_{j} P_{j}(\mu), \sum_{j \in \Xi} h_{j}=1, \quad \varepsilon \ll 1, \frac{\partial \xi(\theta)}{\partial t}=0,
$$

где $h_{j}-$ коэффициенты, определяющие парциальный вклад $j$-й моды в суммарное начальное возмущение; $\Xi-$ множество значений номеров изначально возбужденных мод.

Удовлетворяя начальным условиям для вещественных констант $a_{n}$ и $b_{n}$ в $(19)$, получим значения

$$
a_{n}=\frac{1}{2} h_{j} \delta_{n, j}, \quad b_{n}=0, \quad(j \in \Xi, n=2,3, \ldots),
$$

где $\delta_{n, j}-$ символ Кронекера. Подставляя выражения $a_{n}$ и $b_{n}$ в (19), запишем амплитуды первого порядка малости в выражении для формы поверхности колеблющейся капли в виде

$$
\begin{gathered}
M_{0}(t)=M_{1}(t)=0 \\
M_{n}(t)=\delta_{n, j} h_{j} \cos \left(\omega_{n} t\right), \quad(j \in \Xi, \quad n \geq 2) .
\end{gathered}
$$


Используя эти выражения и переходя к размерным переменным, выпишем отличные от нуля компоненты квадрупольного момента

$$
D_{11}(t)=D_{22}(t)=-\frac{1}{2} D_{33}(t)=-Q R^{2} \varepsilon \delta_{2, j} h_{2} \cos \left(\omega_{2} t\right)
$$

Для того чтобы рассчитать по (1) интенсивность квадрупольного излучения, вычислим третью производную по времени от вышевыписанных диагональных компонент $D_{\alpha \beta}$ и найдем сумму их квадратов, максимальное значение которой запишется в виде

$$
\sum_{\alpha, \beta=1}^{3}\left(\frac{\partial^{3}}{\partial t^{3}} D_{\alpha \beta}(t)\right)_{\max }^{2}=6 Q^{2} R^{4} \varepsilon^{2} h_{2}^{2} \omega_{2}^{6}
$$

Окончательно аналитическое выражение для интенсивности электромагнитного квадрупольного излучения заряженной сферической капли примет вид

$I=\frac{1}{30 c^{5}} Q^{2} R^{4} \varepsilon^{2} h_{2}^{2} \omega_{2}^{6}=\frac{256}{15} \frac{Q^{2} \sigma^{3} \varepsilon^{2} h_{2}^{2}}{c^{5} R^{5} \rho^{3}}\left(1-\frac{Q^{2}}{16 \pi \sigma R^{3}}\right)^{3}$,

где $\omega_{2}$ определено по (18), $h_{2}^{2}-$ коэффициент, определяющий парциальный вклад основной моды в начальное возмущение поверхности капли. Выражение (24) в смысле реализации основных зависимостей интенсивности излучения $I$ от $Q, R, \sigma, \rho$ совпадает с таковыми, рассчитанными для $n=2$ традиционным методом, на основе анализа дисперсионного уравнения [4-5,21]: в асимптотике малого заряда на капле (при $\left.Q^{2} / 16 \pi \sigma R^{3} \ll 1\right)[4,5]$ и в $[21]-$ в асимптотике сильного (близкому к критическому по Рэлею [23]) заряда (при $Q^{2} / 16 \pi \sigma R^{3} \sim 1$ ) [20,22].

Используя (24), можно оценить величину интенсивности фонового шумового электромагнитного излучения от различных жидкокапельных систем искусственного и естественного происхождения, например таких, как конвективные облака. Возможный источник электромагнитного излучения связан с осцилляциями конечной амплитуды мелких капель из диапазона наиболее часто встречающихся в облаке размером от 3 до $30 \mu \mathrm{m}$. Концентрация $n$ таких капель в облаке $\sim 10^{3} \mathrm{~cm}^{-3}$ [23]. Осцилляции большой амплитуды облачных капель могут быть вызваны различными причинами: коагуляцией; дроблением на более мелкие в результате процессов столкновения или в результате реализации электростатической неустойчивости; гидродинамическим и электрическим взаимодействием близко пролетающих капель; аэродинамическим взаимодействием с развитой мелкомасштабной турбулентностью, характерной для грозовых облаков. Амплитуды колебаний облачных капель, согласно данным натурных наблюдений [13-15], могут достигать десятков процентов от радиуса капли.

Проведем оценку интенсивности квадрупольного электромагнитного излучения уединенной слабо заряженной капли, связанного с ее основной модой осцилляций $(j=2)$. Для численных оценок примем значения

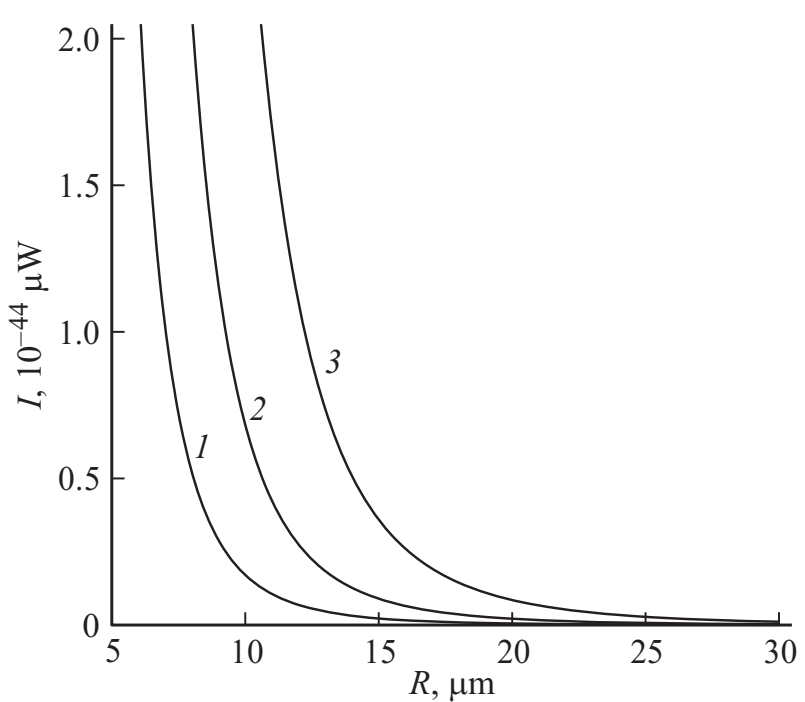

Рис. 1. Зависимости интенсивности электромагнитного квадрупольного излучения единичной заряженной каплей от радиуса равновеликой сферической капли, рассчитанные при $\varepsilon=0.1, h_{2}=1, \sigma=73 \mathrm{dyn} / \mathrm{cm}$. Кривая 1 соответствует $Q=2.5 \cdot 10^{-6} \mathrm{CGSE}\left(\sim 1 \cdot 10^{-3} Q_{k p}\right.$ при $R=3 \mu \mathrm{m}$ и $\sim 3 \cdot 10^{-4} Q_{k p}$ при $\left.R=30 \mu \mathrm{m}\right) ; 2-Q=5 \cdot 10^{-6} \mathrm{CGSE}$ $\left(\sim 3 \cdot 10^{-3} Q_{k p}\right.$ при $R=3 \mu \mathrm{m}$ и $\sim 5 \cdot 10^{-4} Q_{k p}$ при $\left.R=30 \mu \mathrm{m}\right)$; $3-Q=10 \cdot 10^{-6} \mathrm{CGSE}\left(\sim 5 \cdot 10^{-3} Q_{k p}\right.$ при $R=3 \mu \mathrm{m} \quad$ и $\sim 1 \cdot 10^{-3} Q_{k p}$ при $\left.R=30 \mu \mathrm{m}\right)$.

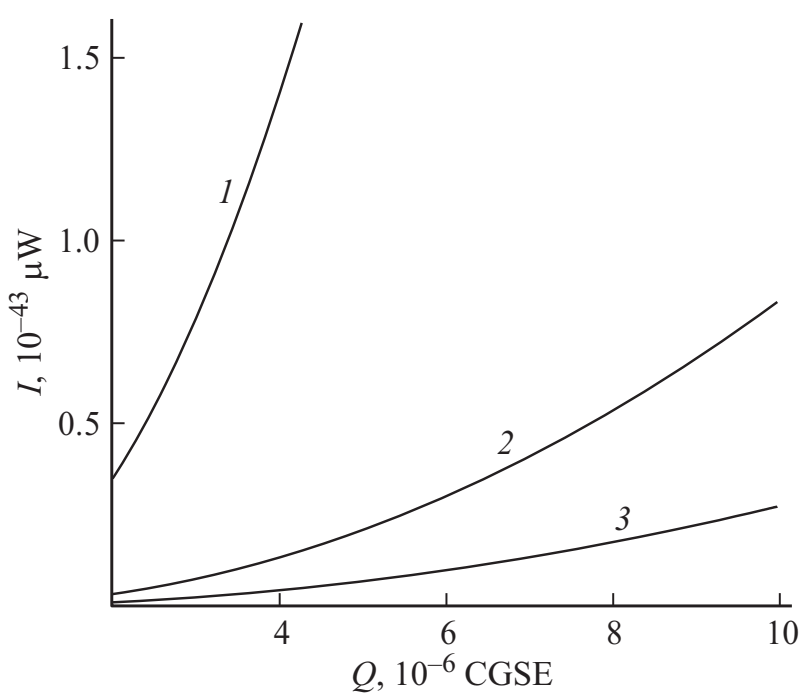

Рис. 2. Зависимости интенсивности электромагнитного квадрупольного излучения единичной заряженной каплей от ее заряда, рассчитанные при тех же значениях $\varepsilon, \rho, \sigma, h_{2}$, что и на рис. $1 . R, \mu \mathrm{m}: 1-5,2-8,3-10$.

физических величин такими же, как в работе [5], в которой исследовалось излучение от осциллирующей заряженной капли методом, предложенным в [1] (на основе анализа дисперсионного уравнения для капли идеальной жидкости), чтобы можно было сравнить результаты расчетов: $\varepsilon=0.1, h_{2}=1, \sigma=73 \mathrm{dyn} / \mathrm{cm}, \rho=1 \mathrm{~g} / \mathrm{cm}^{3}, Q=$ $=2.5 \cdot 10^{-6} \mathrm{CGSE} \quad\left(\sim 1 \cdot 10^{-3} Q k o \quad\right.$ при $\quad R=3 \mu \mathrm{m} \quad$ и 


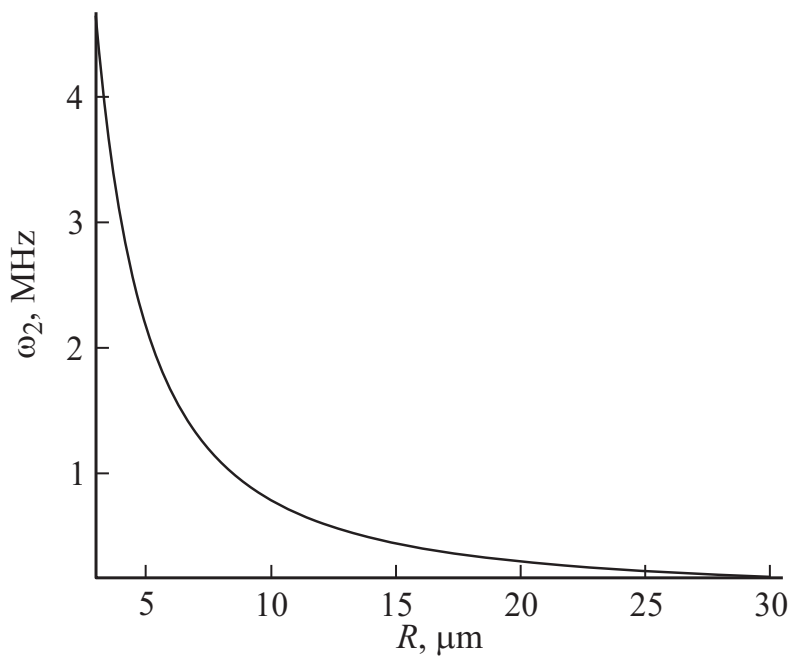

Рис. 3. Зависимость частоты электромагнитного квадрупольного излучения единичной заряженной каплей от радиуса сферической капли, рассчитанная при $Q=2.5 \cdot 10^{-6} \mathrm{CGSE}$ $\left(\sim 1 \cdot 10^{-3} Q_{k p}\right.$ при $R=3 \mu \mathrm{m}$ и $\sim 3 \cdot 10^{-4} Q_{k p}$ при $\left.R=30 \mu \mathrm{m}\right)$ и тех же значениях $\varepsilon, \rho, \sigma, h_{2}$, что и на рис. 1 .

$\sim 3 \cdot 10^{-4} Q_{k p}$ при $R=30 \mu \mathrm{m}$ ). Тогда из (22) при радиусе капли $R=30 \mu \mathrm{m}$ несложно получить $I \sim 7 \cdot 10^{-48} \mu \mathrm{W}$ на частоте $\approx 100 \mathrm{kHz}$. Для капли радиусом $R=8 \mu \mathrm{m}$ получим значение интенсивности $I \sim 5 \cdot 10^{-45} \mu \mathrm{W}$ на частоте $\approx 1 \mathrm{MHz}$. Для капли радиусом $R=3 \mu \mathrm{m}$ получим значение интенсивности излучения $I \sim 7 \cdot 10^{-43} \mu \mathrm{W}$ на частоте $\approx 4.5 \mathrm{MHz}$ (рис. 1). Из проведенных оценок и рис. 1 видно, что с увеличением размера капли интенсивность излучения быстро убывает. При увеличении радиуса на порядок (от $R=3$ до $R=30 \mu \mathrm{m}$ ) интенсивность электромагнитного излучения уменьшается в $10^{5}$ раз.

На рис. 2 приведены характеристики излучения в зависимости от заряда (в предположении его малости) осциллирующей капли, рассчитанные для капель разных размеров. Видно, что с увеличением заряда интенсивность излучения быстро увеличивается: при увеличении заряда в 2 раза интенсивность излучения увеличивается в 4 раза.

На рис. 3 приведена зависимость частоты излучения (частоты осцилляций капли) от радиуса капли в размерном виде. Видно, что с увеличением размера капли частота излучения быстро снижается по закону (18).

Расчеты, проведенные в [5], по методике, предложенной в [1], совпадают с полученными выше и качественно, и количественно. Это свидетельствует о том, что в $[4,5]$ рассчитана интенсивность именно квадрупольного излучения, так как интенсивность дипольного излучения (обнаруживаемого в аналитических расчетах следующего порядка малости по безразмерной амплитуде осцилляций [25]) на 14-15 порядков величины больше.

Следует отметить, что в настоящем анализе и в расчетах [5] брались весьма малые заряды капель, тогда как в общем случае целесообразно рассмотреть и большие вплоть до предельных значений в смысле реализации неустойчивости поверхности капли по отношению к комбинации собственного и индуцированного внешним локальным электрическим полем зарядов. Это следует сделать хотя бы потому, что таковыми становятся условия в момент разряда молнии [25].

Примем в отличие от проведенной выше оценки, что $Q=1.8 \cdot 10^{-3} \mathrm{CGSE}\left(\sim 0.9 Q_{k p}\right.$ при $R=10 \mu \mathrm{m}$

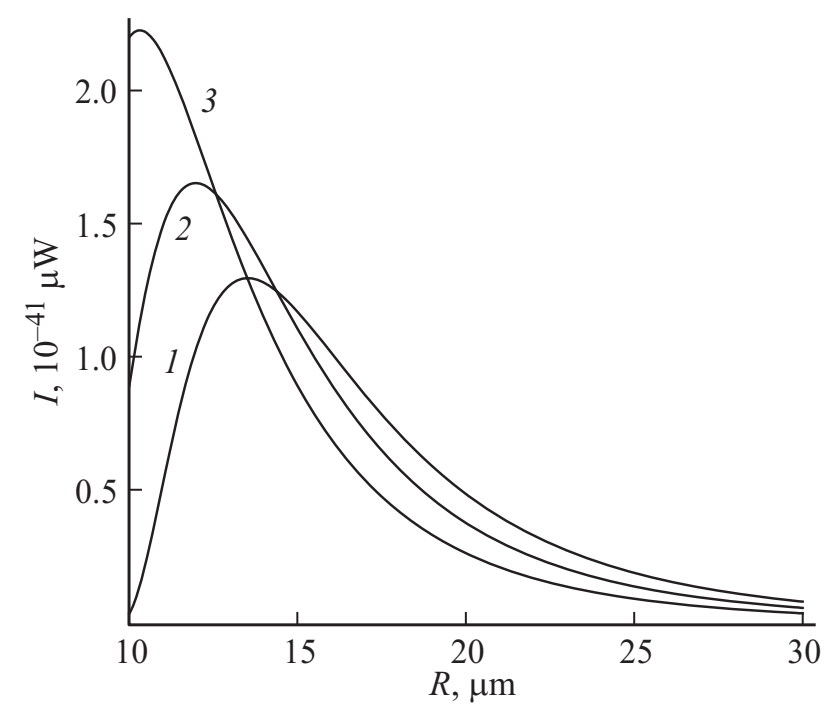

Рис. 4. Зависимости интенсивности электромагнитного квадрупольного излучения единичной сильно заряженной каплей от радиуса равновеликой сферической капли, рассчитанные при тех же значениях $\varepsilon, \rho, \sigma, h_{2}$, что и на рис. 1 . Кривая 1 соответствует $Q=1.8 \cdot 10^{-3} \mathrm{CGSE}$ $\left(\sim 0.9 \cdot 10^{-3} Q_{k p}\right.$ при $R=10 \mu \mathrm{m}$ и $\sim 0.18 Q_{k p}$ при $\left.R=30 \mu \mathrm{m}\right)$; $2-Q=1.5 \cdot 10^{-3} \mathrm{CGSE} \quad\left(\sim 0.87 Q_{k p} \quad\right.$ при $\quad R=10 \mu \mathrm{m} \quad$ и $\sim 0.15 Q_{k p} \quad$ при $\left.\quad R=30 \mu \mathrm{m}\right) ; \quad 3-Q=1.2 \cdot 10^{-3} \mathrm{CGSE}$ $\left(\sim 0.7 Q_{k p}\right.$ при $R=10 \mu \mathrm{m}$ и $\sim 0.12 Q_{k p}$ при $\left.R=30 \mu \mathrm{m}\right)$.

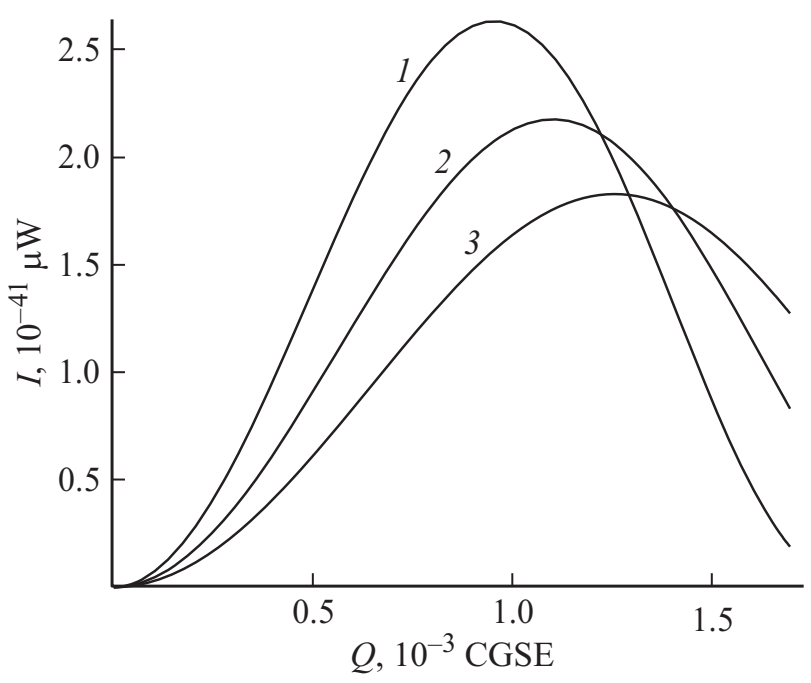

Рис. 5. Зависимости интенсивности электромагнитного квадрупольного излучения единичной заряженной каплей от ее заряда, рассчитанные при тех же значениях $\varepsilon, \rho, \sigma, h_{2}$, что и на рис. $1 . R, \mu$ m: Кривая $1-10,2-11,3-12$. 
и $\sim 0.18 Q_{k p}$ при $\left.R=30 \mu \mathrm{m}\right)$. Из рис. 4 видно, что в этом случае вид зависимости $I \sim I(R)$ качественно изменяется. В частности, при радиусе капли $R=12 \mu$ m несложно получить $I \sim 4 \cdot 10^{-41} \mu \mathrm{W}$ на частоте $\approx 400 \mathrm{kHz}$, а уже при $R=20 \mu \mathrm{m}$ получим значение интенсивности $I \sim 2 \cdot 10^{-41} \mu \mathrm{W}$ на частоте $\approx 300 \mathrm{kHz}$. Для капли радиусом $R=30 \mu \mathrm{m}$ получим значение интенсивности квадрупольного излучения $I \sim 3 \cdot 10^{-42} \mu \mathrm{W}$ на частоте $\approx 100 \mathrm{kHz}$ (рис. 4). Видно, что с увеличением радиуса капли интенсивность излучения быстро растет и, достигнув наибольшего значения, начинает снижаться. Такая зависимость объясняется множителем

$$
\left(1-\frac{Q^{2}}{16 \pi \sigma R^{3}}\right)^{3}
$$

в (24). При малых зарядах капель он мало отличается от единицы, а при стремлении заряда к критическому по Рэлею [22] быстро стремится к нулю. Видно, что в рассматриваемой ситуации наибольший вклад в излучение от облака будут давать капли мелких размеров.

На рис. 5 приведены характеристики излучения в зависимости от заряда осциллирующей капли (при больших значениях заряда), рассчитанные для капель разных размеров. Видно, что искомые зависимости качественно аналогичны приведенным на рис. 4.

Зависимости частоты излучения (частоты осцилляций капли) от радиуса капли при больших зарядах на капле аналогичны приведенным на рис. 3.

Проведенные оценки выполнены для уединенной капли, интенсивность же электромагнитного излучения из облака, состоящего из $N$ капель будет примерно в $N$ раз больше, если принять для оценки по порядку величины, что все капли имеют одинаковый размер и что все капли осциллируют синфазно. Это может иметь место при резком изменении величины электрического поля в облаке (изменение за характерное время, меньшее периода осцилляций капель), что имеет место при разряде молнии. Если капли осциллируют независимо друг от друга с произвольными фазами, то интегральная интенсивность излучения из облака будет больше, чем интенсивность излучения одиночной капли в $\sqrt{N}$ раз. Объемные концентрации капель и распределение их по размерам в облаках различных типов можно найти в [23].

\section{Заключение}

Прямым расчетом найдена интенсивность квадрупольного излучения от заряженной капли, оказавшаяся того же порядка величины, что и интенсивность излучения, найденная на основе косвенных вычислений в [4,5], проведенных по схеме расчета, предложенной в [1]. В соответствии с общей теорией квадрупольное излучение связано с осцилляциями только основной моды, т.е. со сфероидальными осцилляциями поверхности капли.
Работа выполнена при поддержке грантов РФФИ 14-01-00170-а и 14-08-00240-а.

\section{Список литературы}

[1] Калечии, В.И., Нахутин И.Е., Полуэктов П.П. // ДАН CССР. 1982. Т. 262. № 6. С. 1344-1347.

[2] Григорьев А.И., Ширяева С.О., Голованов А.С., Рыбакова М.В. // ЖТФ. 2002. Т. 72. Вып. 1. С. 8-14.

[3] Григорьев А.И., Ширяева С.О. // Изв. РАН. МЖГ. 2002. № 5. C. 67-73.

[4] Богатов Н.А. // Сб. тез. докл. VI Межд. конф. Солнечноземные связи и физика предвестников землетрясений. Петропавловск-Камчатский, 2013. ДВО РАН. С. 10-11.

[5] Grigoryev A.I., Kolbneva N.Yu., Shiryaeva S.O. // Surf. Eng. Appl. Elect. 2015. Vol. 51. N 6. P. 530-539.

[6] Ширяева С.О., Григорьев А.И., Белоножко Д.Ф., Голованов А.С. // Письма в ЖТФ. 2001. Т. 27. Вып. 20. С. 65-71.

[7] Белочерковский А.В., Дивинский Л.И. идр. Активнопассивная радиолокация грозовых и грозоопасных очагов в облаках / Под ред. Л.Г. Качурина и Л.И. Дивинского. СПб.: Гидрометеоиздат, 1992. 286 с.

[8] Качурин Л.Г. Физические основы воздействия на атмосферные процессы. Л.: Гидрометеоиздат, 1990. 463 с.

[9] Грин X., Лейн В. Аэрозоли - пыли, дымы и туманы. Л.: Химия, 1969. $428 \mathrm{c.}$

[10] Амелин А.Г. Теоретические основы образования тумана при конденсации пара. М.: Химия, 1966. 295 с.

[11] Мазин И.П., Хргиан А.Х., Имянитов И.М. Облака и облачная атмосфера. Справочник. Л.: Гидрометеоиздат, 1989. $647 \mathrm{c}$.

[12] Френкель Я.И. // ЖЭТФ. 1936 Т. 6. № 4. С. 348-350.

[13] Beard K.V. // Rev. Geophys. 1987. Vol. 25. N 3. P. 357-370.

[14] Стерлядкин В.В. //. Изв. АН СССР. ФАО. 1988. Т. 24. № 6. C. 613-621.

[15] Beard K.V., Tokay Ali. // Geoph. Res. Lett. 1991. Vol. 18. N 12. P. 2257-2260.

[16] Ландау Л.Д., Лифиии, Е.М. Теория поля. М.: Наука, 1973. $504 \mathrm{c}$.

[17] Левич В.Г. Курс теоретической физики. Т. 1. М.: Наука, 1969. $912 \mathrm{c}$.

[18] Ландау Л.Д., Лифшии, Е.М. Электродинамика сплошных сред. М.: Наука, 1982. 621 с.

[19] Варшалович Д.А., Москалев А.Н., Херсонский В.К. Квантовая теория углового момента. Л.: Наука, 1975. 436 с.

[20] Hendrics C.D., Schneider J.M. // J. Amer. Phys. 1963. Vol. 1. N 6. P. $450-453$.

[21] Григорьев А.И., Колбнева Н.Ю., Ширяева С.О. // ЖТФ. 2016. Т. 86. Вып. 8. С. 68-75.

[22] Rayleigh (Strutt J.W.) // Phil. Mag. 1882. Vol. 14. P. 184-186.

[23] Мазин И.П., Шметер C.M. Облака. Строение и физика образования. Л.: Гидрометеоиздат, 1983. $280 \mathrm{c.}$

[24] Grigor'ev A.I., Shiryaeva S.O. // Phys. Scrip. 1996. Vol. 54. P. 660-666.

[25] Ширяева С.О. // ЖТФ. 2002. Т. 72. Вып. 4. С. 15-19. 